\title{
Secreted semaphorin 5A suppressed pancreatic tumour burden but increased metastasis and endothelial cell proliferation
}

\author{
A Sadanandam ${ }^{*, 1,2,3}$, SS Sidhu ${ }^{4}$, S Wullschleger $^{3}$, S Singh', ML Varney', C-S Yang', AE Ashour ${ }^{5}$, SK Batra $^{6}$ \\ and RK Singh*,1,6
}

'Department of Pathology and Microbiology, University of Nebraska Medical Center, 985900 Nebraska Medical Center, Omaha, NE 68198-5900, USA; ${ }^{2}$ Swiss Institute of Bioinformatics (SIB), Quartier Sorge, Batiment Genopode, Lausanne 1015, Switzerland; ${ }^{3}$ Swiss Institute for Experimental Cancer Research (ISREC), Swiss Federal Institute of Lausanne (EPFL), Station 19, Batiment SV, Lausanne 1015, Switzerland; ${ }^{4}$ Institut National de la Santé et de la Recherche Médicale U753, Laboratoire Immunologie des Tumeurs Humaines, Interaction Effecteurs Cytotoxiques-Système Tumoral, Institut Fédératif de Recherche-54, Villejuif, France; ${ }^{5}$ Department of Pharmacology, College of Pharmacy, King Saud University, Riyadh, Saudi Arabia; ${ }^{6}$ Department of Biochemistry and Molecular Biology, University of Nebraska Medical Center, Omaha, NE 68198, USA

BACKGROUND: Our earlier reports demonstrated that membrane-bound semaphorin 5A (SEMA5A) is expressed in aggressive pancreatic cancer cells and tumours, and promotes tumour growth and metastasis. In this study, we examine whether $(I)$ pancreatic cancer cells secrete SEMA5A and (2) that secreted SEMA5A modulates certain phenotypes associated with tumour progression, angiogenesis and metastasis through various other molecular factors and signalling proteins.

METHODS AND RESULTS: In this study, we show that human pancreatic cancer cell lines secrete the extracellular domain (ECD) of SEMA5A (SEMA5A-ECD) and overexpression of mouse Sema5A-ECD in Pancl cells (not expressing SEMA5A; Pancl-Sema5AECD; control cells - Pancl-control) significantly increases their invasion in vitro via enhanced ERK phosphorylation. Interestingly, orthotopic injection of Panc I-Sema5A-ECD cells into athymic nude mice results in a lower primary tumour burden, but enhances the micrometastases to the liver as compared with Pancl-control cells. Furthermore, there is a significant increase in proliferation of endothelial cells treated with conditioned media (CM) from Pancl-Sema5A-ECD cells and a significant increase in microvessel density in Pancl-Sema5A-ECD orthotopic tumours compared with those from Pancl-control cells, suggesting that the increase in liver micrometastases is probably due to increased tumour angiogenesis. In addition, our data demonstrate that this increase in endothelial cell proliferation by Sema5A-ECD is mediated through the angiogenic molecules - interleukin- 8 and vascular endothelial growth factor.

CONCLUSION: Taken together, these results suggest that a bioactive, secreted form of Sema5A-ECD has an intriguing and potentially important role in its ability to enhance pancreatic tumour invasiveness, angiogenesis and micrometastases.

British Journal of Cancer (2012) I 07, 50 I-507. doi:I0.1038/bjc.20 I2.298 www.bjcancer.com

Published online 10 July 2012

(C) 2012 Cancer Research UK

Keywords: pancreatic cancer; secreted SEMA5A; tumour burden and metastasis; semaphorin; reduced tumour burden; angiogenic factors

Pancreatic adenocarcinoma is one of the leading causes of cancerrelated deaths in the United States. The prognosis of patients suffering from pancreatic adenocarcinoma is extremely poor. More than $80 \%$ of patients suffer from metastatic disease at the time of diagnosis and almost all the patients die either because of the local disease or metastases. The overall survival of pancreatic cancer patients is $<6$ months from the time of diagnosis and current therapeutic strategies are inadequate (Stathis and Moore, 2010). This low survival of pancreatic cancer patients is due to lack of early detection and/or metastatic markers. This urges for a better understanding of the molecular mechanism(s) behind pancreatic adenocarcinoma tumour progression and metastasis to identify novel metastatic markers for the disease.

Semaphorins are a family (containing eight subfamilies) of axonal growth molecules that contain secreted, glycosylphospatidylinositol-anchored and transmembrane signalling proteins. They

\footnotetext{
*Correspondence: Dr A Sadanandam; E-mail: Anguraj.sadanandam@ epfl.ch or Dr RK Singh; E-mail: rsingh@unmc.edu

Received 6 February 2012; revised 6 June 2012; accepted 12 June 2012; published online 10 July 2012
}

share a conserved $\sim 500$ amino acids semaphorin domain in their $\mathrm{N}$-terminal regions and have subfamily-specific domains in their carboxy-terminal regions (Chen et al, 1998; Yazdani and Terman, 2006). They were originally identified as bi-functional axonal growth molecules involved in the development of the nervous system (Chen et al, 1998). Semaphorins exert their cellular functions by binding to two different families of proteins called plexins and neuropilins (Kolodkin et al, 1993; Chen et al, 1998; Yazdani and Terman, 2006; Roth et al, 2009). In addition to their function during normal physiological processes such as the development of the nervous, cardiac, skeletal and vascular systems as well as the immune response, semaphorins also have major roles during pathogenesis of tumour formation, angiogenesis and metastasis (Chen et al, 1998; Christensen et al, 1998; Brambilla et al, 2000; Tamagnone and Comoglio, 2000; Kusy et al, 2005; Roth et al, 2009; Sadanandam et al, 2010b). Among the different semaphorins, the role of membrane-bound semaphorins in cancer has recently drawn more attention.

Previously, we identified semaphorin 5A (SEMA5A), a transmembrane bound semaphorin, as a putative metastatic marker for pancreatic cancer (Sadanandam et al, 2007). Semaphorin $5 \mathrm{~A}$ is 
characterised by five thrombospondin-1-specific repeats in its extracellular domain (ECD) in addition to the semaphorin and plexin domains (Adams et al, 1996). Semaphorin 5A is known to induce inhibitory responses during optic nerve development (Oster et al, 2003) and also has major roles in innate immunity (Sugimoto et al, 2006) and idiopathic autism (Melin et al, 2006). For the first time, we observed the expression of SEMA5A in human pancreatic tumours, but not in normal pancreatic tissue. In addition, we showed that SEMA5A is expressed by pancreatic cancer cell lines established from metastases, but not those from primary tumours. We also demonstrated the membrane localisation of SEMA5A particularly to regions of cell-cell adhesion in aggressive pancreatic cancer cell lines. Overexpression of membrane-bound mouse Sema5A in pancreatic cancer cells has been demonstrated to increase migration and aggregation in vitro and tumorigenesis and metastasis in vivo (Sadanandam et al, 2010b). These results suggest that membrane-bound SEMA5A functions as a paracrine molecule involved in pancreatic tumour growth and metastasis. However, different reports on various cancer types either endogenously expressing SEMA5A or treated with the ECD of mouse Sema5A have contrasting tumour phenotypes ( $\mathrm{Li}$ and Lee, 2010; Lu et al, 2010; Pan et al, 2010).

Recently, others and we have demonstrated the interaction of Sema5A with Plexin B3 in different mouse tissues and various cell lines (ectopically expressing Plexin B3; Artigiani et al, 2004; Sadanandam et al, 2008). Moreover, we have demonstrated that Sema5A is a proangiogenic molecule that regulates various steps of angiogenesis including endothelial cell proliferation, survival and migration in vitro and sprouting of blood vessels in vivo through its functional receptor, Plexin B3 (Sadanandam et al, 2010a). However, SEMA4D, a membrane-bound protein and a close relative with structural similarity to SEMA5A (Sadanandam et al, 2007), has been shown to be secreted (Wang et al, 2001) and can regulate tumour angiogenesis and metastasis (Basile et al, 2006). In this report, we demonstrate that pancreatic cancer cells secrete ECD of SEMA5A (SEMA5A-ECD) and that secreted Sema5A enhances invasiveness and metastasis of tumour cells via ERK phosphorylation and proliferation of endothelial cells through upregulation of angiogenic factors.

\section{MATERIALS AND METHODS}

\section{Cell culture}

Human pancreatic cancer cell lines (Panc1 and T3M4) and immortalised human dermal microvascular endothelial cells (HMEC-1; obtained from the Centre for Disease Control and Prevention, Atlanta, GA, USA; Ades et al, 1992) were maintained in culture as an adherent monolayer with RPMI-1640/DMEM with 5\% Foetal Calf Serum (Mediatech, Herndon, VA, USA) supplemented with $1 \mathrm{X}$ nonessential amino acids, $2 \mathrm{~mm}$ L-glutamine and $1 \mathrm{X}$ vitamin solution.

\section{Transfection of pancreatic cancer cells and purification of recombinant protein}

Pancl cells were stably transfected with either $1 \mu \mathrm{g}$ of a cDNA construct containing the mouse Sema5A-ECD conjugated to human IgG $\mathrm{F} c \gamma$ cloned in to the pcDNA3.1 vector (generous gift from Dr David Stretavan, University of California San Francisco at San Francisco, CA, USA) or pcDNA3.1 control vector (Stratagene, La Jolla, CA, USA) using LipofectAMINE (Invitrogen, Carlsbad, CA, USA) according to the protocol provided by the manufacturer. Pancl cells transfected with Sema5A-ECD (Panc1-Sema5A-ECD) or control vector (Panc1-control) were selected and maintained with $400 \mu \mathrm{g} \mathrm{ml}^{-1} \mathrm{G} 418$ sulphate (Mediatech). Fc-tagged Sema5A-ECD was isolated by protein-A affinity chromatography from the supernatant of Panc1-Sema5A-ECD expressing cells and the protein purity was tested using silver staining (Fischer Chemical, Fairlawn, NJ, USA) as described previously (Sadanandam et al, 2010a).

\section{Tumorigenic and metastatic assays}

The tumorigenic and metatstatic assays were performed using male athymic BALB/c nude mice (NCI-nu, 6-8 weeks old, purchased from the National Cancer Institute). In accordance with approval from the American Association of Laboratory Animal Care and current regulations and standards of the US Department of Agriculture, Department of Health and Human Services and National Institute of Health, the mice used for this study were maintained under specific pathogen-free conditions in facilities. All procedures performed in mice were in accordance with the institutional guidelines and were approved by the University of Nebraska Medical Center Institution Animal Care and Use Committee.

For tumour growth and metastasis, the preparation of cells and the orthotopic injections in to the pancreas of the mice were performed as described in our previous study (Sadanandam et al, 2010b).

\section{Immunohistochemistry (IHC)}

Fixed paraffin-embedded tissue samples on slides were deparafinised in a series of xylene and alcohol treatments (two times xylenes, one time each in $100 \%, 95 \%, 80 \%$ and $70 \%$ alcohol) for $5 \mathrm{~min}$ each. The slides were washed with PBS for $5 \mathrm{~min}$ for three times and quenched for endogenous peroxidase activity by incubating with $3 \% \mathrm{H}_{2} \mathrm{O}_{2}$ in PBS for $5 \mathrm{~min}$. Slides were again washed thoroughly with PBS and blocked with blocking buffer (10\% horse/donkey serum) for $30 \mathrm{~min}$. Without further washing, the samples were incubated with primary antibody (anti-PCNA $\left(5 \mu \mathrm{g} \mathrm{ml}^{-1}\right.$, Santa Cruz Biotechnology, Santa Cruz, CA, USA), antiSEMA5A ( $5 \mu \mathrm{g} \mathrm{ml}^{-1}$; Sadanandam et al, 2010b), anti-interleukin (IL)$8\left(2 \mu \mathrm{g} \mathrm{ml}^{-1}\right)$, anti-vascular endothelial growth factor (VEGF; $2 \mu \mathrm{g} \mathrm{ml}^{-1}$; R\&D System, Minneapolis, MN, USA) or anti-CD31 ( $1: 100$, Abcam, Cambridge, MA, USA) in blocking buffer for overnight at $4{ }^{\circ} \mathrm{C}$ and then with biotinylated secondary antibody for $30 \mathrm{~min}$ to $1 \mathrm{~h}$ after washing with PBS. After washing the slides in $\mathrm{PBS}$, the samples were incubated with $\mathrm{ABC}$ reagent (Vector Laboratories, Burlingame, CA, USA) for 15-30 min. Finally, DAB substrate (Vector Laboratories) was added until a brown colour developed and counter stained with hematoxylin. The quantitation of the number of cells stained for a particular antibody was determined by counting at least five different random fields in the same section at $\times 200$ resolution using a Nikon microscope (Nikon Inc., Melville, NY, USA). The average number of positive cells was calculated.

\section{mRNA analysis}

Total cellular RNA isolation and reverse transcription based polymerase chain reaction (RT-PCR) specific to SEMA5A and $\beta$-actin was performed as described (Sadanandam et al, 2010b).

\section{In vitro proliferation assay}

For endothelial cell proliferation, cells $\left(1 \times 10^{3}\right)$ were seeded in individual wells of 96-well flat-bottomed plates in triplicate and treated with serum-free supernatant from Panc1-Sema5A-ECD or Panc1-control cells. The proliferative activity of the cells after $72 \mathrm{~h}$ of incubation was measured using the 3-(4,5-dimethylthiazol-2-yl)-2,5diphenyltetrazolium bromide (MTT) assay using a microtiter plate reader (Bio-Tek Instruments, Inc., Winooski, VT, USA) at $570 \mathrm{~nm}$ as described (Sadanandam et al, 2010a).

\section{Invasion and migration assay}

For invasion assay, cells $\left(1 \times 10^{5}\right)$ were plated onto the upper chamber of transwell chambers and coated with matrigel $(6.5 \mathrm{~mm}$; 
Corning Costar Corp., Cambridge, MA, USA) on the bottom of the upper chamber. MTT was added to the wells during the last $2 \mathrm{~h}$ of incubation. Using a cotton swab, the residual cells from the top of the transwell chambers were removed. The inserts were transferred to a carrier plate and dimethyl sulfoxide was added to solubilise the crystals. The migratory activity was measured by reading at $570 \mathrm{~nm}$ using a microplate reader.

For migration assay using MEK1 inhibitor (PD98059, Millipore, Billerica, MA, USA), cells $\left(1 \times 10^{5}\right)$ along with $10 \mu \mathrm{M}$ MEK1 inhibitor or media alone were plated onto the upper chamber of transwell chambers and media on the bottom chamber. The cells were incubated for $4 \mathrm{~h}$ at $37^{\circ} \mathrm{C}$ and cells that did not pass through the membrane pores were removed using a cotton swab. Migrated cells were stained using the Hema 3 kit (Fisher Scientific Company L.L.C., Kalamazoo, MI, USA) as per the manufacturer's instructions. Cells were counted in 10 random fields $(\times 200)$ and expressed as the average number of cells per field of view. The data are represented as the average of three independent experiments.

\section{Immunoprecipitation and western blot analysis}

Cells $\left(1 \times 10^{6}\right)$ were plated on 10 -cm cell culture dishes and serumstarved for $24 \mathrm{~h}$. Serum-free supernatants or conditioned media (CM) and lysates from T3M4 cells were collected, concentrated using Amicon Ultra-30 Centrifugal Filter Units (Millipore) and were directly subjected to western blot as described (Sadanandam et al, 2010b).

For immunoprecipiation, CM from Panc1-Sema5A-ECD or control cells were collected and were immunoprecipitated using Protein A/G (Invitrogen) and anti-SEMA5A antibody and incubated at $4{ }^{\circ} \mathrm{C}$ overnight. Later, the immunoprecipitates were washed three times using RIPA buffer followed by SDS-PAGE and western blot using anti-SEMA5A antibody as described (Sadanandam et al, 2008).

For ERK phosphorylation, pancreatic cancer cells were seeded in $10-\mathrm{cm}$ cell culture dishes. After the cells reached $80-90 \%$ confluence, cells were treated with Sema5A-ECD recombinant protein $\left(10 \mathrm{ng} \mathrm{ml}^{-1}\right)$ for 5 or $30 \mathrm{~min}$ or media alone. Cells were washed three times with cold PBS and lysed in membrane lysis buffer and the lysates (20 $\mu \mathrm{g}$ perlane) were separated on SDS-PAGE and subjected to western blot as described (Sadanandam et al, 2010b). Densitometric quantitation of intensity of the bands was performed using the ImageQuant (GE Healthcare, Piscataway, NJ, USA).

\section{Enzyme-linked immunosorbant assay (ELISA)}

IL-8 or VEGF protein levels in culture supernatants were determined using enzyme-linked immunosorbent assay (ELISA) matched-pair antibodies for IL-8 (Endogen Inc., Woburn, MA, USA) or VEGF (R\&D Systems) according to the manufacturer's instructions with modification as described previously (Varney et al, 2003). Briefly, $50 \mu \mathrm{l}$ of culture supernatant from treated or untreated cells was assayed in duplicate using the sandwich ELISA technique. A standard curve of the absorbance $v s$ the concentration of protein (IL-8 or VEGF) in the standard wells was generated. We determined the concentration of protein in the unknown samples by comparing the absorbance of the sample to the standard curve.

\section{Statistical analysis}

The significance of the data was determined by the Student's $t$-test (two-tailed) for all the studies. $P<0.05$ was deemed significant. All statistical analyses were done using the SPSS software (SPSS, Inc., Chicago, IL, USA).

\section{RESULTS}

\section{Secretion of SEMA5A in pancreatic cancer cells}

To examine whether pancreatic cancer cells secrete SEMA5A, we performed western blots for SEMA5A using serum-free supernatant (CM, see Materials and Methods for supernatant preparation) and protein lysates collected from T3M4, a pancreatic cancer cell line expressing high levels of SEMA5A (Sadanandam et al, 2010b). The results shown in Figure 1A demonstrate a band at $\sim 110 \mathrm{kD}$ in the T3M4 CM whereas the protein lysate shows bands at $\sim 135 \mathrm{kD}$ (membrane-bound SEMA5A) and $\sim 110 \mathrm{kD}$ (equivalent to the molecular weight of its extraceullar domain). These results suggest that SEMA5A is secreted in pancreatic adenocarcinoma cell lines.

\section{Overexpression of Sema5A-ECD in pancreatic cancer cells increases their invasive potential}

As the $\sim 110-\mathrm{kD}$ band in T3M4 supernatant (Figure 1A) is predicted to be equivalent to SEMA5A-ECD, we studied the role of SEMA5A-ECD in pancreatic cancer. We stably transfected Panc1 cells, which do not express SEMA5A, with a cDNA construct containing mouse Sema5A-ECD (Panc1-Sema5A-ECD) fused with human IgG Fc $\gamma$ region at the C-terminus or with empty vector (Panc1-control). Panc1-Sema5A-ECD cells expressed Sema5A mRNA whereas Panc1-control cells did not show detectable levels of Sema5A mRNA (Figure 1Ba). In addition, we performed immunoprecipitation followed by western blot both using antiSEMA5A antibody and supernatant from Panc1-Sema5A-ECD and Panc1-control. We observed a band between $\sim 180$ and $\sim 220 \mathrm{kD}$ that represents the Sema5A-ECD plus with human IgG Fc $\gamma$ region (Figure 1Bb) similar to that shown by Oster et al (2003).

Next, we examined whether expression of Sema5A-ECD in pancreatic cancer cells modulates their invasive potential. Significantly more Panc1-Sema5A-ECD cells invaded through Matrigel as compared with control cells $(P<0.05)$ in the absence of serum (Figure 1C). This result demonstrates that Sema5A-ECD modulates the invasive potential of pancreatic cancer cells.

\section{Expression and phosphorylation of ERK in Sema5A treated Pancl cells}

Downregulation of D-Sema5c has been shown to decrease phosphorylation of ERK in a Drosophila model for metastasis (Woodhouse et al, 2003). To identify a signalling network mediating migration of pancreatic cancer cells by secreted SEMA5A, we examined ERK phosphorylation after treatment with recombinant Sema5A-ECD. Increased levels of p42 ERK phosphorylation were observed in Panc1 cells treated with $10 \mathrm{ng} \mathrm{ml}^{-1}$ of Sema5A-ECD for 5 and $30 \mathrm{~min}$ compared with cells treated with media alone (Figures 1D and E) as well as a slight increase in the level of p44 ERK phosphorylation (Figure 1D and Supplementary Figure 1A). To verify if ERK signalling is important for SEMA5A-ECD induced migration of pancreatic cancer cells, we performed transwell chamber migration assays by treating Panc1-Sema5A-ECD and Panc1-control cells with $10 \mu \mathrm{M}$ concentration of MEK1 inhibitor (PD98059) or media alone. A significant decrease in migration of Panc1-Sema5A-ECD cells with MEK1 inhibitor was observed compared with the treatment with media alone whereas there was not much change in the Panc1control cells (Figure 1F). This data supports the hypothesis that Sema5A-dependent cellular migration is mediated through the increased ERK phosphorylation.

\section{Sema5A-ECD overexpression enhances spontaneous metastasis}

To examine the role of Sema5A-ECD in tumour cells in an orthotopic pancreatic microenvironment, we injected 

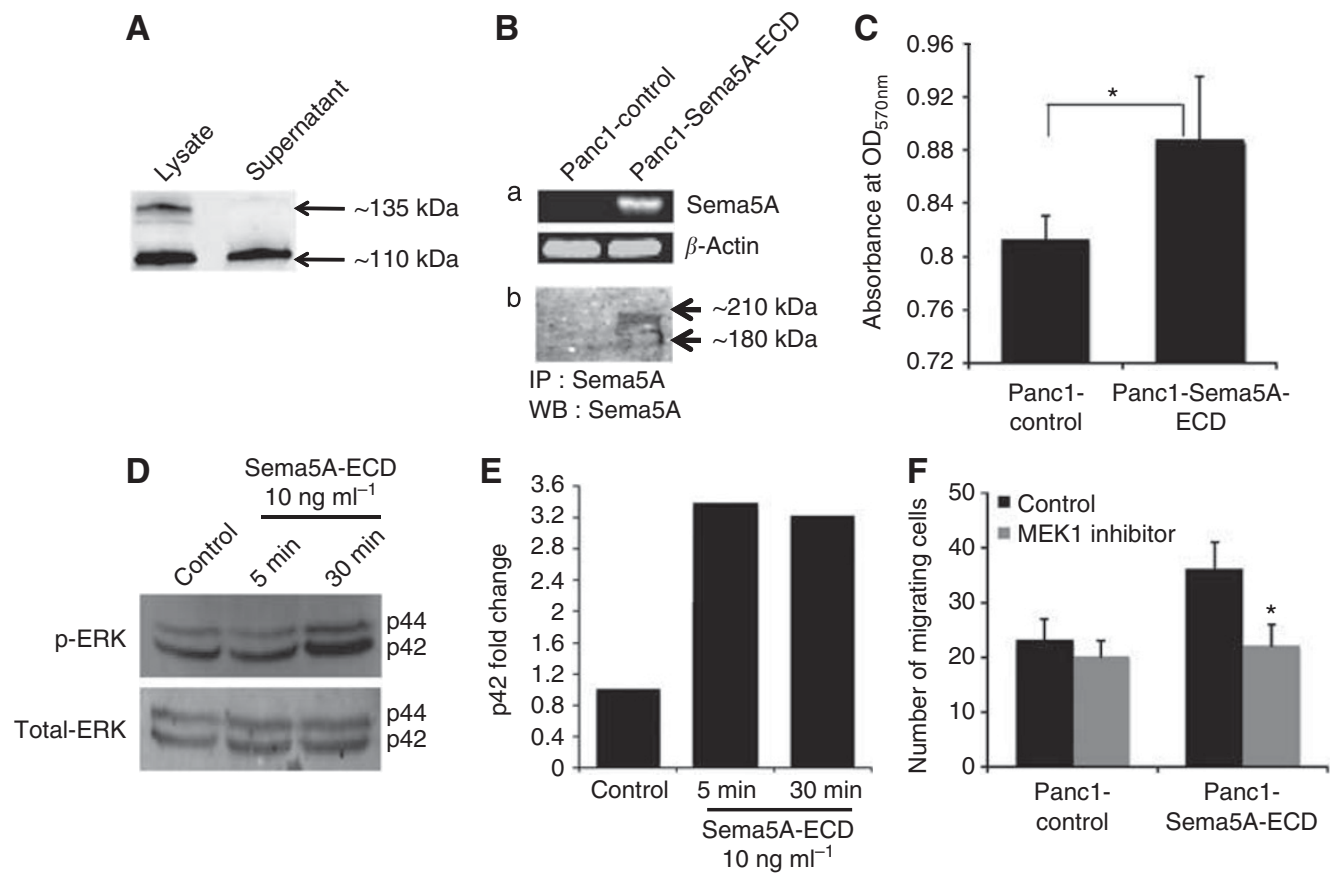

Figure I Secretion of SEMA5A and its invasive role in pancreatic cancer cells. (A) A representative western blot analysis showing expression of membrane-bound SEMA5A in protein lysates and secretion of SEMA5A in supernatant of human pancreatic cancer cell line - T3M4. (B) A standard (a) RT-PCR (using specific primers) and (b) immunoprecipitation and western blot analysis (using anti-SEMA5A antibody) of Pancl cells ectopically expressing mouse Sema5A-ECD (Pancl-Sema5A-ECD) and control vector (Pancl-control) showing expression of Sema5A. $\beta$-Actin was used as an internal control for RT-PCR. (C) Cellular invasion in Pancl-Sema5A-ECD or Pancl-control after I h was measured using Matrigel-coated transwell chambers and MTT assay. Invasion was measured as mean optical density $(O D)$ at $570 \mathrm{~nm}\left(\mathrm{OD}_{570 \mathrm{~nm}}\right) \pm$ standard deviation (s.d., bars) of triplicate culture and *a statistical significance of $P<0.05$. (D) Western blot analysis of phosphorylated and total ERK in protein lysates of Pancl cells treated with $10 \mathrm{ng} \mathrm{ml}^{-1}$ of recombinant Sema5A-ECD protein at different time points $(5$ or $30 \mathrm{~min}$ ) or media alone using appropriate antibodies. (E) Densitometric quantitation of phosphorylated p44 ERK that was normalised to total p44 ERK from western blots from (D) using the ImageQuant software. (F) Cellular migration in Pancl-Sema5A and Pancl-control after $4 \mathrm{~h}$ was measured after treating the cells either with I0 $\mu \mathrm{M}$ MEKI inhibitor (PD98059) or media alone (control) using uncoated transwell chambers and the number of migrated cells was counted at $\times 10$ magnification using a Nikon microscope. The values are number of migrated cells \pm s.d. (bars) of triplicate culture and *a statistical significance of $P<0.05$ using Student $t$-test.

Panc1-Sema5A-ECD or Panc1-control $\left(5 \times 10^{5}\right)$ cells into the pancreas of athymic nude mice. Mice were killed after 10 weeks. The presence of primary tumour in the pancreas and metastatic cells in different organs was confirmed by H\&E staining (data not shown). Surprisingly, no difference in tumour incidence was observed between mice injected with Panc1-Sema5A-ECD or Panc1-control cells (Figure 2A). However, in mice injected with Panc1-Sema5A-ECD cells, tumour burden was lower and yet there was an increased incidence of micrometastases to the liver (Figure 2A). Though there were no obvious macrometastases in either Panc1-Sema5A-ECD mice or control mice, there was a significant increase in number of micrometastatic cells to the liver (Figure 2B). To examine the proliferation of orthotopic tumours, we examined PCNA staining in Panc1-Sema5A-ECD and control tumours by IHC analysis using an anti-PCNA antibody and then counting the number of positive cells. No significant difference in PCNA staining was observed between Panc1-Sema5A-ECD and control tumours (Supplementary Figure 1B).

Next, we performed IHC for CD31 marker for microvessels in orthotopic tumours. Here, we identified a significant increase in microvessel density in orthotopic tumours expressing Sema5AECD as compared with control tumours (Figures 2C and D). These data suggest that Sema5A-ECD stimulates aggressiveness in pancreatic tumour cells leading to increased micrometastases via increased tumour angiogenesis, but with lower primary tumour burden.

\section{Sema5A-ECD increases endothelial cell proliferation} and upregulates expression of pro-angiogenic factors

To investigate whether Sema5A-ECD mediates increased metastasis by increasing endothelial cell proliferation (one of the steps involved in tumour angiogenesis), we performed MTT proliferation assays after treating HMEC-1 endothelial cells with conditioned serum-free supernatants from Panc1-Sema5A-ECD or Panc1-control cells (Figure 3A). Significantly increased proliferation was observed in HMEC-1 cells treated with serum-free conditioned supernatant from Panc1-Sema5A-ECD as compared with that from Panc1-control cells (Figure 3A). This result demonstrates that Sema5A-ECD increases proliferation of endothelial cells.

Next, we examined the expression of well-known pro-angiogenic regulators IL-8 and VEGF in the serum-free supernatants of Panc1Sema5A-ECD or control cells in vitro by ELISA. Interleukin-8 and VEGF were significantly increased in the supernatant of Panc1Sema5A-ECD cells as compared with control cells (Figures 3B and $\mathrm{C}$ ). We further validated the regulation of IL-8 and VEGF expression by Sema5A-ECD by treating Panc1-Sema5A-ECD cells with neutralising antibody to Sema5A for $72 \mathrm{~h}$ and then measuring the expression levels of IL-8 and VEGF in the supernatant using ELISA. A significant decrease $(P<0.05)$ in IL- 8 and VEGF protein expression was seen in the supernatant of Panc1-Sema5A-ECD treated with $10 \mathrm{ng}$ of neutralising antibody to Sema5A (Figures 3D and E). Upregulation of IL-8 expression was also seen in 
Secreted SEMA5A in pancreatic cancer

A

\begin{tabular}{|c|c|c|c|c|}
\hline \multicolumn{2}{|c|}{} & & \multicolumn{2}{|c|}{ Incidence of } \\
\hline Cell lines & $\begin{array}{c}\text { Average weight } \\
\text { of mice in g }\end{array}$ & $\begin{array}{c}\text { Average weight } \\
\text { of tumour in g }\end{array}$ & Primary tumour & $\begin{array}{c}\text { Micrometastases to } \\
\text { liver }\end{array}$ \\
\hline Panc1-control & $24.68 \pm 3.34$ & $0.77 \pm 0.33$ & 100 & 60 \\
\hline $\begin{array}{c}\text { Panc1-Sema5A- } \\
\text { ECD }\end{array}$ & $24.966 \pm 2.52$ & $0.31 \pm 0.15$ & 100 & 100 \\
\hline
\end{tabular}
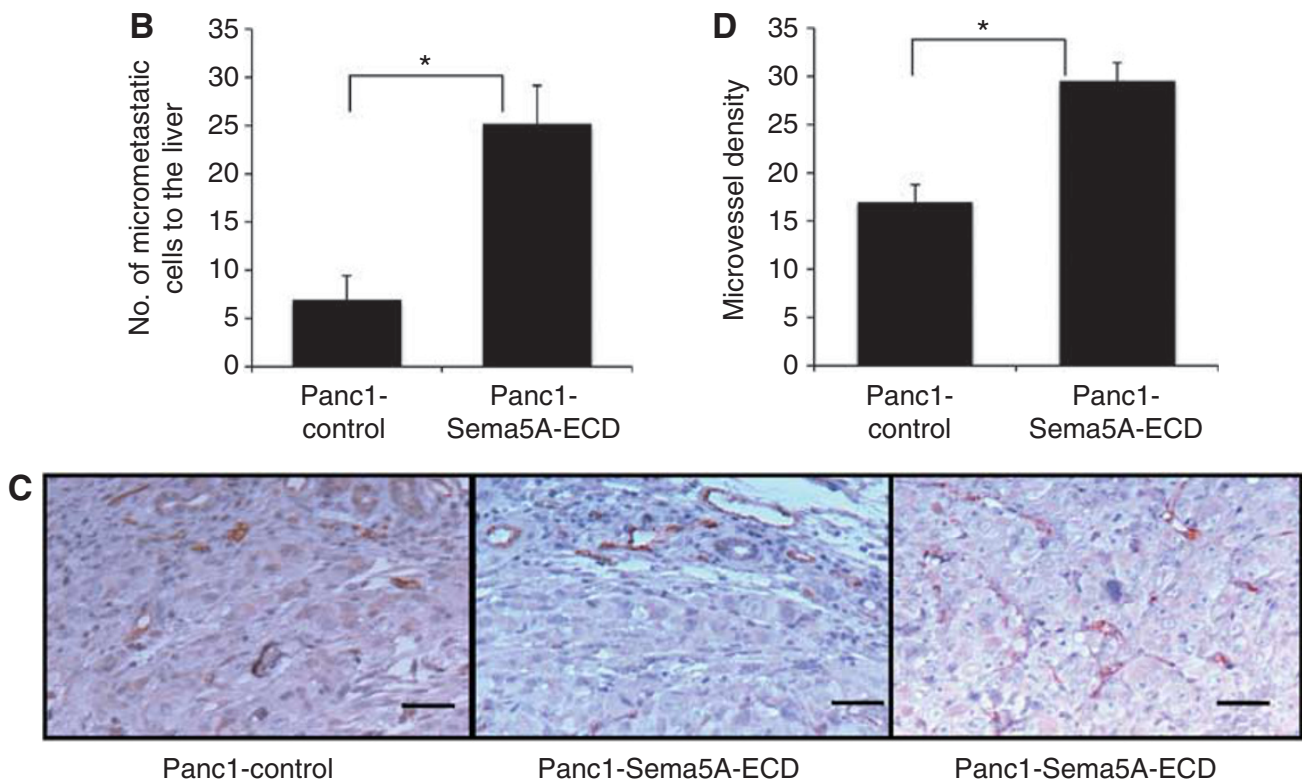

Figure 2 Orthotopic assay, micrometastasis and microvessel density of Panc-I Sema5A-ECD. Athymic nude mice $(n=5)$ were injected orthotopically with Pancl-control or Pancl-Sema5A-ECD and were euthanised after 10 weeks. (A) Average weight of mice and tumour (tumour burden) as well as incidence of primary tumour and metastasis to the liver is shown. (B) There are a significantly enhanced number of micrometastatic cells per field of $\times 200$ resolution of the Nikon microscope in the liver in Panc-I-Sema5A-ECD tumour-bearing mice as compared to control mice. (C) Immunohistochemistry using CD3I staining showing microvessels in Pancl-Sema5A-ECD and control orthotopic tumours. There is an increased angiogenesis in Pancl-Sema5AECD compared to the Pancl-control orthotopic tumours. (D) Densitometric quantitation of microvessel density from C. per field at $\times 200$ resolution of the Nikon microscope. The values are number of microvessels \pm s.d. (bars) of five different areas per field or different slides and *a statistical significance of $P<0.05$.

Panc1-Sema5A-ECD orthotopic tumours as compared with control tumours by IHC $(P<0.05$; Figures $3 \mathrm{~F}$ and $\mathrm{G})$. However, we did not observe a significant change in VEGF staining between orthotopic tumours from Panc1-Sema5A-ECD and Panc1-control (Supplementary Figure 1C). These results demonstrate that Sema5A-ECD increases the protein expression of IL-8 and to a certain extent VEGF in pancreatic cancer cells and tumours.

\section{DISCUSSION}

Previously, we found that pancreatic tumours and aggressive pancreatic cancer cell lines express membrane-bound SEMA5A, however, it is not expressed in normal pancreatic tissues or less aggressive pancreatic cancer cell lines (Sadanandam et al, 2007, 2010b). In this study, we sought to determine if pancreatic cancer cells secrete SEMA5A and whether secreted SEMA5A has a role in pancreatic tumour progression, metastasis and angiogenesis by regulating other molecules.

Many secreted forms of membrane-associated proteins such as CD44 (Stickeler et al, 2000) and transforming growth factor- $\beta$ type III receptor (Bandyopadhyay et al, 2002) were reported to be involved in tumour metastasis. One transmembrane protein of the semaphorin family, SEMA4D, which displays structural similarity to SEMA5A (Sadanandam et al, 2007), has also been reported to be released as a functional secreted protein and has been shown to be involved in tumorigenesis and metastasis (Wang et al, 2001; Basile et al, 2004, 2006). In this report, we detected SEMA5A protein in the serum-free supernatant of a human pancreatic cancer cell line (T3M4) and the $\sim 110-\mathrm{kD}$ band was predicted to be similar to that of the Sema5A-ECD. To our knowledge, this is the first report showing that SEMA5A is secreted.

In our previous study, we demonstrated that membrane-bound Sema5A increases pancreatic cancer invasion in vitro and metastasis in vivo (Sadanandam et al, 2010b). In this report, we studied the role of secreted SEMA5A in pancreatic tumour progression and metastasis by overexpressing mouse Sema5AECD in Panc1 cells not expressing SEMA5A. Sema5A-ECD expression in pancreatic cancer cells demonstrated increased invasion in vitro. This increase in invasion of pancreatic cancer cells induced by Sema5A-ECD especially in serum-free conditions suggests that this molecule is a motogenic factor secreted by tumour cells.

Previously, we have shown that orthotopic injections of pancreatic cancer cells overexpressing membrane-bound Sema5A in athymic nude mice are tumorigenic and metastatic (Sadanandam et al, 2010b). Here, we demonstrate that orthotopic injection of Sema5A-ECD-expressing cells resulted in reduced tumour burden, but enhanced micrometastases to the liver as compared with control transfected cells. It has already been reported that small primary tumours could lead to metastasis in different cancer types (Menz et al, 1985; Sho et al, 1998; Chiang 
A

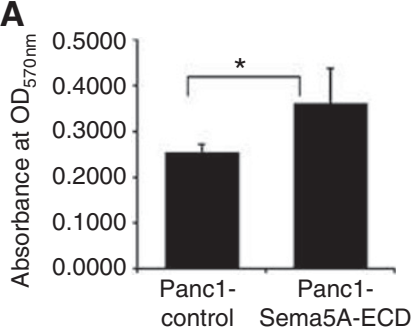

B

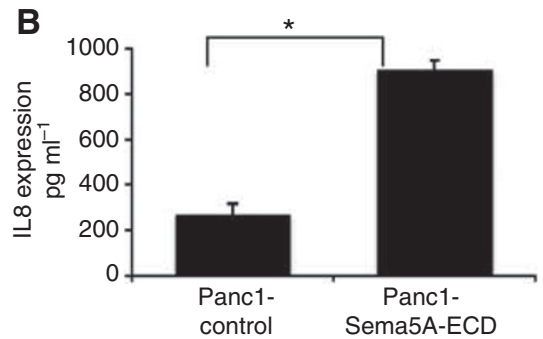

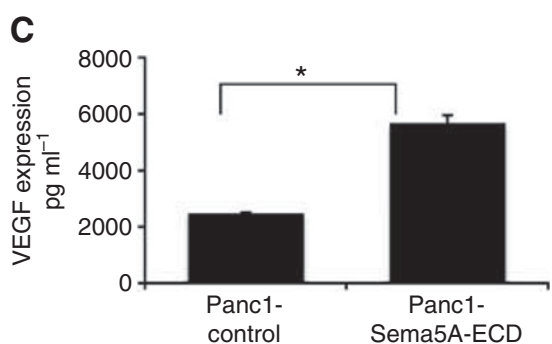

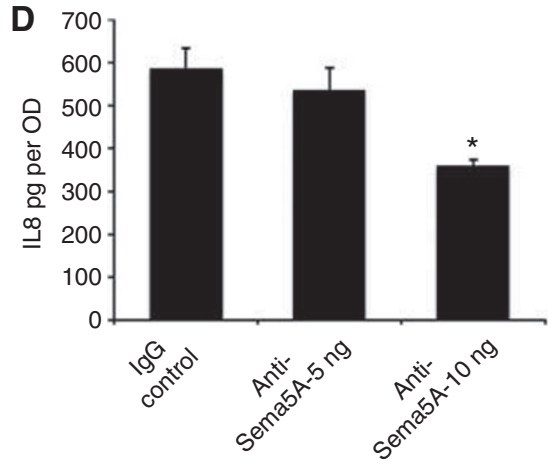

F

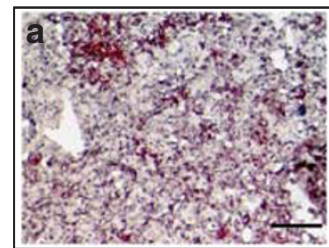

Panc1-control

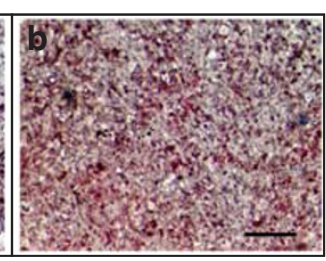

Panc1-Sema5A-ECD
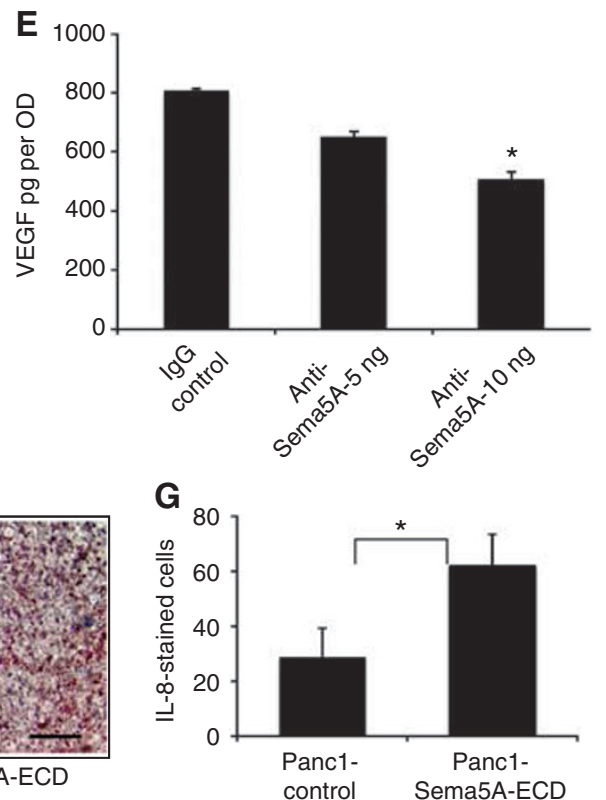

Figure 3 Sema5A-ECD stimulates endothelial cell proliferation and increases the expression of angiogenic factors. (A) MTT proliferation assay after $72 \mathrm{~h}$ using 1000 HMEC-I cells per well that were treated with conditioned medium (no serum) from Pancl-Sema5A-ECD or Pancl-control. There is a significant $(P<0.05)$ increase in proliferation of HMEC-I in the presence of supernatant from Pancl-Sema5A-ECD cells compared with control cells with no serum. Proliferation was measured as mean optical density $(O D)$ at $570 \mathrm{~nm}\left(\mathrm{OD}_{570 \mathrm{~nm}}\right) \pm$ s.d. (bars) of triplicate culture and *statistical significance of $P<0.05$. Enzyme-linked immunosorbent assay for (B) IL-8 and (C) VEGF protein in the supernatant of Pancl-Sema5A-ECD and Pancl-control cells using appropriate antibodies. There is increased expression of IL-8 and VEGF in the supernatants of Pancl-Sema5A-ECD compared with control. Data are mean pg mg- I \pm s.e.m. (bars) of triplicate samples and *statistical significance of $P<0.05$. Neutralisation of Sema5A-ECD in supernatants of Pancl-Sema5A-ECD using $10 \mathrm{ng} \mathrm{ml}^{-1}$ of anti-Sema5A or rabbit-lgG antibody followed by ELISA for (D) IL-8 or (E) VEGF. There is a significant decrease in IL-8 and VEGF using $10 \mathrm{ng} \mathrm{ml}^{-9}$ of anti-Sema5A antibody compared with rabbit-lgG. Data are mean pg/OD \pm s.d. (bars) of triplicate samples and * statistical significance of $P<0.05$. (F) Immunohistochemistry showing IL-8 staining in (a) Pancl-control and (b) Pancl-Sema5A-ECD orthotopic tumours using anti-IL-8 antibody. The magnification is $\times 200$ resolution using a Nikon microscope. (G) The number of cells positive for IL-8 from $\mathbf{F}$. The values are mean number of cells \pm s.d. (bars) of three slides. *Statistical significant difference of $P<0.05$.

et al, 2008). The mechanism by which secreted Sema5A induces increased metastasis with reduced tumour burden is intriguing. Based on our previous findings that Panc1 cells express Plexin B3, a functional receptor for SEMA5A (Sadanandam et al, 2008), we suggest that the secretion of SEMA5A in pancreatic cancer induces an autocrine mechanism via the ERK phosphorylation to metastasise to distant organs but with smaller primary tumour, unravelling this cell signalling mechanism might be the basis of our future studies.

We have previously shown that Sema5A can promote angiogenesis, in general (Sadanandam et al, 2010a). In this study, we show that Panc1-Sema5A-ECD is biologically active and also has the ability to promote tumour angiogenesis as shown by its ability to significantly increase in vitro proliferation of HMEC-1 endothelial cells and significantly increase microvessel density in orthotopic Panc1-Sema5A-ECD tumours compared with control tumours. In addition, supernatants from Panc1-Sema5A-ECD cells show increased expression of pro-angiogenic factors, IL-8 and VEGF.
We speculate that secreted Sema5A derived from the membranebound protein could be the reason for the increased IL-8 and VEGF expression. The direct effect of Sema5A-ECD on endothelial cells to secrete IL-8 and VEGF was shown using neutralising antibody against Sema5A in endothelial cells. Overall, the increase in micrometastasis in Sema5A-ECD-expressing pancreatic cancer cells is due to increased tumour angiogenesis via IL-8 and VEGF.

In conclusion, in this study, for the first time we show that malignant pancreatic cancer cells secrete SEMA5A and that this secreted form demonstrates the ability to drive micrometastatic spread via the enhancement of angiogenesis and migration of pancreatic cancer cells via increased ERK signalling and expression of angiogenic factors, but with reduced primary tumour burden. These results suggest a potentially important and hitherto unsuspected role of the secreted form of SEMA5A in pancreatic cancer metastasis. Further study is warranted to examine the presence of secreted SEMA5A in pancreatic cancer patients and to understand the autocrine mechanism of its action. 


\section{ACKNOWLEDGEMENTS}

This work was supported in part by the Molecular Therapeutics Program, Nebraska Department of Health and Human Services and by Grants - CA72781 (RKS); Grants U54CA163120 (SKB), and Cancer Centre Support Grant (P30CA036727) from the National Cancer Institute, National Institutes of Health.

Supplementary Information accompanies the paper on British Journal of Cancer website (http://www.nature.com/bjc)

\section{REFERENCES}

Adams RH, Betz H, Puschel AW (1996) A novel class of murine semaphorins with homology to thrombospondin is differentially expressed during early embryogenesis. Mech Dev 57: 33-45

Ades EW, Candal FJ, Swerlick RA, George VG, Summers S, Bosse DC, Lawley TJ (1992) HMEC-1: establishment of an immortalized human microvascular endothelial cell line. J Invest Dermatol 99: 683-690

Artigiani S, Conrotto P, Fazzari P, Gilestro GF, Barberis D, Giordano S, Comoglio PM, Tamagnone L (2004) Plexin-B3 is a functional receptor for semaphorin 5A. EMBO Rep 5: 710-714

Bandyopadhyay A, Zhu Y, Malik SN, Kreisberg J, Brattain MG, Sprague EA, Luo J, Lopez-Casillas F, Sun LZ (2002) Extracellular domain of TGFbeta type III receptor inhibits angiogenesis and tumor growth in human cancer cells. Oncogene 21: 3541-3551

Basile JR, Barac A, Zhu T, Guan KL, Gutkind JS (2004) Class IV semaphorins promote angiogenesis by stimulating Rho-initiated pathways through plexin-B. Cancer Res 64: 5212-5224

Basile JR, Castilho RM, Williams VP, Gutkind JS (2006) Semaphorin 4D provides a link between axon guidance processes and tumor-induced angiogenesis. Proc Natl Acad Sci USA 103: 9017-9022

Brambilla E, Constantin B, Drabkin H, Roche J (2000) Semaphorin SEMA3F localization in malignant human lung and cell lines: a suggested role in cell adhesion and cell migration. Am J Pathol 156: 939-950

Chen H, He Z, Tessier-Lavigne M (1998) Axon guidance mechanisms: semaphorins as simultaneous repellents and anti-repellents. Nat Neurosci 1: 436-439

Chiang WF, Liu SY, Fang LY, Lin CN, Wu MH, Chen YC, Chen YL, Jin YT (2008) Overexpression of galectin-1 at the tumor invasion front is associated with poor prognosis in early-stage oral squamous cell carcinoma. Oral Oncol 44: 325-334

Christensen CR, Klingelhofer J, Tarabykina S, Hulgaard EF, Kramerov D, Lukanidin E (1998) Transcription of a novel mouse semaphorin gene, $\mathrm{M}$-semaH, correlates with the metastatic ability of mouse tumor cell lines. Cancer Res 58: 1238-1244

Kolodkin AL, Matthes DJ, Goodman CS (1993) The semaphorin genes encode a family of transmembrane and secreted growth cone guidance molecules. Cell 75: 1389-1399

Kusy S, Nasarre P, Chan D, Potiron V, Meyronet D, Gemmill RM, Constantin B, Drabkin HA, Roche J (2005) Selective suppression of in vivo tumorigenicity by semaphorin SEMA3F in lung cancer cells. Neoplasia 7: 457-465

Li X, Lee AY (2010) Semaphorin 5A and plexin-B3 inhibit human glioma cell motility through RhoGDIalpha-mediated inactivation of Rac1 GTPase. J Biol Chem 285: 32436-32445

$\mathrm{Lu}$ TP, Tsai MH, Lee JM, Hsu CP, Chen PC, Lin CW, Shih JY, Yang PC, Hsiao CK, Lai LC, Chuang EY (2010) Identification of a novel biomarker, SEMA5A, for non-small cell lung carcinoma in nonsmoking women. Cancer Epidemiol Biomarkers Prev 19: 2590-2597

Melin M, Carlsson B, Anckarsater H, Rastam M, Betancur C, Isaksson A, Gillberg C, Dahl N (2006) Constitutional downregulation of SEMA5A expression in autism. Neuropsychobiology 54: 64-69

Menz J, Sterrett G, Wall L (1985) Metastatic basal cell carcinoma associated with a small primary tumour. Australas J Dermatol 26: 121-124
Oster SF, Bodeker MO, He F, Sretavan DW (2003) Invariant Sema5A inhibition serves an ensheathing function during optic nerve development. Development 130: 775-784

Pan G, Lv H, Ren H, Wang Y, Liu Y, Jiang H, Wen J (2010) Elevated expression of semaphorin $5 \mathrm{~A}$ in human gastric cancer and its implication in carcinogenesis. Life Sci 86: 139-144

Roth L, Koncina E, Satkauskas S, Cremel G, Aunis D, Bagnard D (2009) The many faces of semaphorins: from development to pathology. Cell $\mathrm{Mol}$ Life Sci 66: 649-666

Sadanandam A, Rosenbaugh EG, Singh S, Varney M, Singh RK (2010a) Semaphorin $5 \mathrm{~A}$ promotes angiogenesis by increasing endothelial cell proliferation, migration, and decreasing apoptosis. Microvasc Res 79: 1-9

Sadanandam A, Varney ML, Kinarsky L, Ali H, Mosley RL, Singh RK (2007) Identification of functional cell adhesion molecules with a potential role in metastasis by a combination of in vivo phage display and in silico analysis. Omics 11: 41-57

Sadanandam A, Varney ML, Singh RK (2008) Identification of semaphorin $5 \mathrm{~A}$ interacting protein by applying apriori knowledge and peptide complementarity related to protein evolution and structure. Genomics Proteomics Bioinformatics 6: 163-174

Sadanandam A, Varney ML, Singh S, Ashour AE, Moniaux N, Deb S, Lele SM, Batra SK, Singh RK (2010b) High gene expression of semaphorin $5 \mathrm{~A}$ in pancreatic cancer is associated with tumor growth, invasion and metastasis. Int J Cancer 127: 1373-1383

Sho M, Adachi M, Taki T, Hashida H, Konishi T, Huang CL, Ikeda N, Nakajima Y, Kanehiro H, Hisanaga M, Nakano H, Miyake M (1998) Transmembrane 4 superfamily as a prognostic factor in pancreatic cancer. Int J Cancer 79: 509-516

Stathis A, Moore MJ (2010) Advanced pancreatic carcinoma: current treatment and future challenges. Nat Rev Clin Oncol 7: 163-172

Stickeler E, Vogl FD, Denkinger T, Mobus VJ, Kreienberg R, Runnebaum IB (2000) Soluble CD44 splice variants and pelvic lymph node metastasis in ovarian cancer patients. Int J Mol Med 6: 595-601

Sugimoto M, Fujikawa A, Womack JE, Sugimoto Y (2006) Evidence that bovine forebrain embryonic zinc finger-like gene influences immune response associated with mastitis resistance. Proc Natl Acad Sci USA 103: 6454-6459

Tamagnone L, Comoglio PM (2000) Signalling by semaphorin receptors: cell guidance and beyond. Trends Cell Biol 10: 377-383

Varney ML, Li A, Dave BJ, Bucana CD, Johansson SL, Singh RK (2003) Expression of CXCR1 and CXCR2 receptors in malignant melanoma with different metastatic potential and their role in interleukin-8 (CXCL-8)mediated modulation of metastatic phenotype. Clin Exp Metastasis 20: 723-731

Wang X, Kumanogoh A, Watanabe C, Shi W, Yoshida K, Kikutani H (2001) Functional soluble CD100/Sema4D released from activated lymphocytes: possible role in normal and pathologic immune responses. Blood 97: 3498-3504

Woodhouse EC, Fisher A, Bandle RW, Bryant-Greenwood B, Charboneau L, Petricoin III EF, Liotta LA (2003) Drosophila screening model for metastasis: Semaphorin $5 c$ is required for $1(2) \mathrm{gl}$ cancer phenotype. Proc Natl Acad Sci USA 100: 11463-11468

Yazdani U, Terman JR (2006) The semaphorins. Genome Biol 7: 211

This work is published under the standard license to publish agreement. After 12 months the work will become freely available and the license terms will switch to a Creative Commons Attribution-NonCommercial-Share Alike 3.0 Unported License. 